\title{
微波加热催化反应低温制备 $\boldsymbol{\beta}-\mathrm{SiC}$ 粉体
}

\author{
王军凯，张远卓，李俊怡，张海军，李发亮，韩 否，宋述鹏 \\ (武汉科技大学 省部共建耐火材料与冶金国家重点实验室，武汉 430081)
}

摘 要: 以硅粉和酚醛树脂为原料, 硝酸镍为催化剂前驱体, 采用微波加热催化反应法, 在流通氩气气氛中 $1150^{\circ} \mathrm{C} / 0.5 \mathrm{~h}$ 反应后合成了 $\beta-\mathrm{SiC}$ 粉体。研究了反应温度、催化剂用量和保温时间等对合成 $\beta-\mathrm{SiC}$ 的影响。采用 XRD、 SEM 和 TEM 对产物的物相组成及显微结构进行了表征。结果表明: 微波加热条件下, 无催化剂存在时, $\beta$-SiC 的完 全合成温度为 $1250^{\circ} \mathrm{C}$; 而添加 $1.0 \mathrm{wt} \%$ 的 $\mathrm{Ni}$ 作催化剂时, $1150^{\circ} \mathrm{C} / 0.5 \mathrm{~h}$ 反应后即可合成纯相的 $\beta-\mathrm{SiC}$ 。所合成的试样 中都存在着颗粒状和晶须状两种 $\mathrm{SiC}$, 加入催化剂后会使试样中 $\beta$-SiC 晶须的长径比变大。密度泛函理论(DFT)计 算结果表明, $\mathrm{Ni}-\mathrm{Si}$ 合金纳米颗粒的形成使 $\mathrm{Si}$ 原子之间的键长拉长，弱化了 $\mathrm{Si}$ 原子之间的结合强度，进而促进了 $\mathrm{Si}$ 粉在低温下的碳化反应。

关 键 词: $\beta-\mathrm{SiC}$; 微波加热; 催化反应; 密度泛函理论

中图分类号: TB35 文献标识码: A

\section{Low Temperature Catalytic Synthesis of $\beta$-SiC Powders via Microwave Heating}

WANG Jun-Kai, ZHANG Yuan-Zhuo, LI Jun-Yi, ZHANG Hai-Jun, LI Fa-Liang, HAN Lei, SONG Shu-Peng (The State Key Laboratory of Refractories and Metallurgy, Wuhan University of Science and Technology, Wuhan 430081, China)

\begin{abstract}
SiC powders were synthesized by a microwave heating method at $1150^{\circ} \mathrm{C}$ for 30 min under $\mathrm{Ar}$ atomosphere using silicon powders and phenolic resin as raw materials, and nickel nitrate as catalyst precursor. The effects of temperature, catalyst content and holding time on the formation of SiC were investigated. XRD, SEM and TEM were used to characterize the phase and microstructure of the prepared samples. It indicated that $\beta$-SiC can be synthesized at $1150^{\circ} \mathrm{C}$ for $0.5 \mathrm{~h}$ by using $1.0 \mathrm{wt} \% \mathrm{Ni}$ as catalysts. In contrast, for the sample prepared without $\mathrm{Ni}$ catalysts, corresponding preparation temperature of $\beta$-SiC was as high as $1250^{\circ} \mathrm{C}$. Both $\beta$-SiC whiskers and particles were formed in the samples prepared with or without catalysts, and the length of formed $\beta-\mathrm{SiC}$ whiskers in the samples prepared with $\mathrm{Ni}$ catalysts were larger than those without catalyst. Density functional theory (DFT) calculation shows that the formation of Ni-Si alloy can elongate the length of $\mathrm{Si}-\mathrm{Si}$ chemical bond, weaken its bond strength, and finally accelerate the carbonization reaction of Si powder at a relative low temperature.
\end{abstract}

Key words: $\beta-\mathrm{SiC}$; microwave heating; catalytic-reaction; density functional theory

碳化硅具有熔点高、硬度高、高温强度大、抗＼cjkstart蠕变性能好、热膨胀系数小及热传导率高等优点，

收稿日期: 2016-09-26; 收到修改稿日期：2016-11-13

基金项目: 国家自然科学基金(51472184, 51472185); 湖北省教育厅高等学校优秀中青年科技创新团队计划(T201602) National Natural Science Foundation of China(51472184, 51472185); Program for Innovative Teams of Outstanding Young and Middle-aged Researchers in the Higher Education Institutions of Hubei Province(T201602)

作者简介: 王军凯(1988-), 男, 博士研究生. E-mail: 960331748@qq.com

通讯作者: 张海军, 教授. E-mail: zhanghaijun@wust.edu.cn 
因而在陶瓷、复合材料、耐磨材料及催化等领域有 着广泛应用前景。碳化硅的工业制备方法主要采用 艾奇逊法，该法以石英砂和石油焦为主要原料，具有 原料来源广、成本低等优点。但是, 该方法存在反应 温度高(约 $2400^{\circ} \mathrm{C}$ ), 反应时间长, 产物粒径大, 需要 通过研磨等工艺进行二次处理等缺点 ${ }^{[1]}$ 。

微波加热技术已经广泛用于高温反应制备陶瓷 粉体 ${ }^{[2-9]}$ 。与传统的加热技术相比, 微波加热可以大 幅降低加热温度、减少反应时间; 在分子水平上实 现均匀加热; 加热时能量作用在整个被加热体上, 内外同时受热，对热扩散依赖小，快速、高效、节能、 省时、无污染 ${ }^{[10]}$ 。卢斌等 ${ }^{[11]}$ 以单质硅粉和酚醛树脂 为原料, 采用微波加热工艺, 经 $1300 \sim 1400^{\circ} \mathrm{C}$ 反应 0.5 2 h 后合成了碳化硅纳米线。本课题组 ${ }^{[12]}$ 以正硅 酸乙酯和蔗糖为原料, 采用溶胶一凝胶、微波碳热还 原法, 经 $1300^{\circ} \mathrm{C}$ 反应 $1 \mathrm{~h}$ 得到纯相的碳化硅。郝斌 等 ${ }^{[13]}$ 以乙炔炭黑和硅粉为原料, 采用微波加热工艺, 经 $1100^{\circ} \mathrm{C}$ 反应 $30 \mathrm{~min}$ 或 $1200^{\circ} \mathrm{C}$ 反应 $15 \mathrm{~min}$ 后制备 了纯相的碳化硅粉体。

已有研究还表明, 引入催化剂可以显著促进碳 化硅的合成并改变其形貌。间晓燕等 ${ }^{[14]}$ 以酚醛树脂 为碳源、正硅酸乙酯为硅源、硝酸锆为催化剂制备 了碳化硅粉体, 结果显示, 氧化锆的引入为碳化硅 的生长提供了有利的生长点。Wang 等 ${ }^{[15]}$ 以硝酸铁 为催化剂制备了周期孪晶结构的碳化硅纳米线。徐 武军等 ${ }^{[16]}$ 以正硅酸乙酯及聚乙烯吡咯烷酮为反应 前驱体, 以硝酸铁为催化剂, 采用溶胶-凝胶、碳热 还原法制备了塔状碳化硅纳米棒。研究表明, 催化 剂的存在可显著提高碳化硅的产率。

基于此, 本工作以硅粉及酚醛树脂为原料, 以 硝酸镍为催化剂, 采用微波加热催化反应法合成碳 化硅粉体, 研究了反应温度、催化剂用量和反应时 间等对碳化硅合成的影响, 并根据密度泛函理论 (DFT)的计算结果, 提出了 $\mathrm{Ni}$ 催化剂低温催化 $\mathrm{Si}$ 粉 合成 $\mathrm{SiC}$ 的机理。

\section{1 实验方法}

\section{1 实验原料}

实验用原料有: 热固性酚醛树脂(2221, 武汉力 发公司, 黏度 $=10 \sim 30 \mathrm{~Pa} \cdot \mathrm{s}$, 固含量 $\geqslant 75 \mathrm{wt} \%$, 残碳 率 $\geqslant 42 \mathrm{wt} \%$, 游离酚 $\leqslant 10 \mathrm{wt} \%$ ，水分 $\leqslant 5 \mathrm{wt} \%$, $\mathrm{pH}=6.0 \sim 7.5) 、 \mathrm{Si}$ 粉 $(<2 \mu \mathrm{m}) 、$ 硝酸镍 $\left(\mathrm{Ni}\left(\mathrm{NO}_{3}\right)_{2} \cdot 6 \mathrm{H}_{2} \mathrm{O}\right.$, 分析纯)及无水乙醇等。

\section{2 样品制备}

样品的制备过程如下：1) 先将 $\mathrm{Ni}\left(\mathrm{NO}_{3}\right)_{2} \cdot 6 \mathrm{H}_{2} \mathrm{O}$ 溶于 $30 \mathrm{~mL}$ 无水乙醇, 再将溶液滴加入到 $30 \mathrm{~g}$ 硅粉 中 $(\mathrm{Ni} / \mathrm{Si}=0.5 \mathrm{wt} \% 、 1.0 \mathrm{wt} \% 、 1.5 \mathrm{wt} \%$ 和 $2.0 \mathrm{wt} \%)$, 静 置 $24 \mathrm{~h}$ 后, 放入 $80^{\circ} \mathrm{C}$ 干燥箱中干燥 $12 \mathrm{~h}$, 而后在氩 气气氛下 $600^{\circ} \mathrm{C}$ 热处理 $1 \mathrm{~h}$ 得到表面负载有催化剂的 硅粉;2) 将负载有催化剂的硅粉研磨后与 $100 \mathrm{~g}$ 酚醛 树脂均匀混合, 先 $80^{\circ} \mathrm{C}$ 固化 $12 \mathrm{~h}$, 再 $120^{\circ} \mathrm{C}$ 固化 $24 \mathrm{~h}$, 而后将固化后的样品置于氩气气氛下 $900^{\circ} \mathrm{C}$ 保温 $2 \mathrm{~h}$ 预处理；3) 预处理后的样品采用微波加热炉 (HAMiLab-V3000, $3 \mathrm{~kW}, 2.45 \mathrm{GHz}$ ，长沙隆泰微波 热工有限公司)升温至 $1000 \sim 1300^{\circ} \mathrm{C}$, 并分别保温 10、20 和 $30 \mathrm{~min}$ 。

\section{3 样品表征}

采用 $\chi$ 'pert Pro 型 $\mathrm{X}$ 射线衍射仪(PANalytical, NETHERLANDS, $40 \mathrm{kV}, 40 \mathrm{~mA}$ )分析产物的物相组 成。采用场发射扫描电子显微镜(FE-SEM, Nova400NanoSEM, PHILIPS, NETHERLANDS, $15 \mathrm{kV}$ ) 观察产物的形貌，并用能谱仪(EDS, Ie 350 Penta FEI X-3)分析其元素组成; 采用透射电子显微镜(TEM, JEM-2100UHRSTEM, JEOL, JAPAN, 200 kV)研究产 物的显微结构。

\subsection{DFT 计算}

采用 $\mathrm{Si}_{55}$ 纳米团簇、 $\mathrm{Si}_{43} \mathrm{Ni}_{12}$ 和 $\mathrm{Si}_{37} \mathrm{Ni}_{18}$ 合金纳米 团簇作为计算模型，使用 $\mathrm{Dmol}^{3}$ 程序包进行 DFT 计 算。电子交换和相关能利用广义梯度近似(Generalized gradient approximation, GGA)泛函 PBE 在包含相对论 效应双数值极化函数基组 DNP 水平上进行计算。数 值积分精度采用每个原子约 1000 个格点, 轨道截断 值为 $0.45 \mathrm{~nm}$ 。自洽场 $(\mathrm{SCF})$ 计算收敛标准为电子密度 变化小于 $0.00001 \mathrm{eV}$, 轨道占据使用 thermal smearing $(0.1 \mathrm{eV})$ 方法加速 SCF 计算收敛。在 PBE/DNP 理论 水平上对上述原子团簇的结构进行全优化计算, 具 体参数如下: 能量的收玫标准为 $2 \times 10^{-5} \mathrm{au}$, 力参数 收玫标准为 $4.0 \times 10^{-3} \mathrm{au} / 0.1 \mathrm{~nm}$, 最大位移收玫标准 为 $0.0005 \mathrm{~nm}$ 。分别计算了 $\mathrm{Si}_{55}$ 纳米团簇、 $\mathrm{Si}_{43} \mathrm{Ni}_{12}$ 和 $\mathrm{Si}_{37} \mathrm{Ni}_{18}$ 合金纳米团簇的内聚能变化, 以及这些纳 米团簇(111)面上边缘位置 $\mathrm{Si}-\mathrm{Si}$ 键的键长。

\section{2 结果与讨论}

\section{1 无催化剂时，反应温度对合成碳化硅的影响}

图 1 为不含催化剂的样品在不同温度下反应 $0.5 \mathrm{~h}$ 后的 XRD 图谱, 从图中可知, $1000^{\circ} \mathrm{C}$ 反应 $0.5 \mathrm{~h}$, 试样 


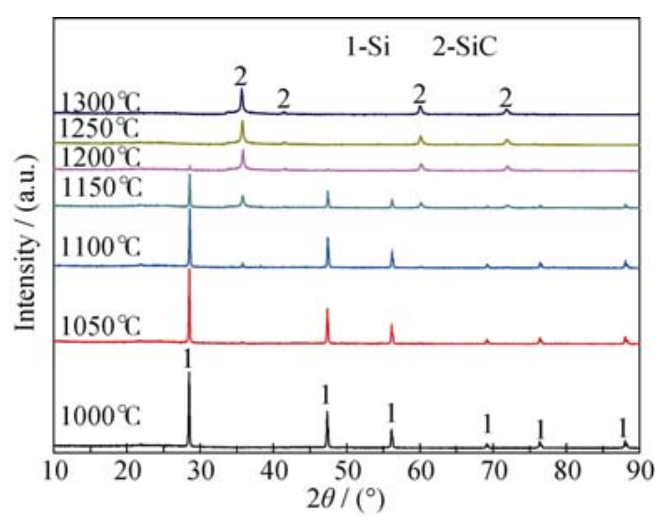

图 1 无催化剂时, 不同温度下反应 $0.5 \mathrm{~h}$ 后试样的 XRD 图谱 Fig. 1 XRD patterns of samples reacted at various temperatures for $0.5 \mathrm{~h}$ without any catalysts

(Si: JCPDS 01-077-2109; SiC: JCPDS 01-073-1708)

XRD 图谱中未发现碳化硅的衍射峰, 说明碳化反应 尚未开始进行; $1050^{\circ} \mathrm{C}$ 反应 $0.5 \mathrm{~h}$, 试样 XRD 图谱中 出现了微弱的碳化硅衍射峰, 说明碳化反应刚刚开 始进行; $1100^{\circ} \mathrm{C}$ 反应 $0.5 \mathrm{~h}$, 试样 XRD 图谱中碳化硅 的衍射峰有所增强; $1150^{\circ} \mathrm{C}$ 反应 $0.5 \mathrm{~h}$, 试样 XRD 图 谱中碳化硅的衍射峰进一步增强; $1200^{\circ} \mathrm{C}$ 反应 $0.5 \mathrm{~h}$, 产物中金属硅的衍射峰强度明显下降, 表明此时大 部分硅粉已转变为碳化硅; $1250^{\circ} \mathrm{C}$ 反应 $0.5 \mathrm{~h}$, 试样 XRD 图谱中仅有碳化硅的衍射峰, 表明硅粉已全部 参与碳化反应。与常规加热合成 $\mathrm{SiC}$ 所需温度 $1500^{\circ} \mathrm{C}$ 相比 ${ }^{[1,17]}$, 采用微波加热可以使 $\mathrm{SiC}$ 的合成 温度降低约 $250^{\circ} \mathrm{C}$ 。

\section{2 以 $\mathrm{Ni}$ 为催化剂时, 反应温度对合成碳化硅的 影响}

图 2 为添加 $2 \mathrm{wt} \% \mathrm{Ni}$ 做催化剂时, 不同温度下硅 粉碳化反应后的 XRD 图谱, 从图中可知, $1000^{\circ} \mathrm{C}$ 反 应 $0.5 \mathrm{~h}$, 试样 XRD 图谱中开始出现微弱的碳化硅 的衍射峰, 说明碳化反应已经开始进行。与图 1 对 比可知, 催化剂镍的存在明显促进了碳化硅的生 成。随着反应温度从 $1000^{\circ} \mathrm{C}$ 升高至 $1100^{\circ} \mathrm{C}, \mathrm{XRD}$ 图 谱中碳化硅的衍射峰不断增强; 当反应温度继续升 高至 $1150^{\circ} \mathrm{C}$ 时, XRD 图谱中金属硅的衍射峰完全消 失, 表明此时硅粉已完全发生碳化反应。继续升高 温度, 产物的物相组成无变化。与不加催化剂的结 果(图 1)对比可知, 催化剂 $\mathrm{Ni}$ 的加入使硅粉的完全 碳化反应温度降低了约 $100^{\circ} \mathrm{C}$ 。

\section{3 催化剂加入量对合成碳化硅的影响}

催化剂 $\mathrm{Ni}$ 加入量不同时, $1150^{\circ} \mathrm{C}$ 碳化 $0.5 \mathrm{~h}$ 试样 的 XRD 图谱如图 3 所示。当催化剂加入量为 $0.5 \mathrm{wt} \%$ 时, 试样 XRD 图谱中就有很强的 $\beta-\mathrm{SiC}$ 的衍射峰, 说 明硅粉的碳化反应已经开始; 当催化剂用量增加到

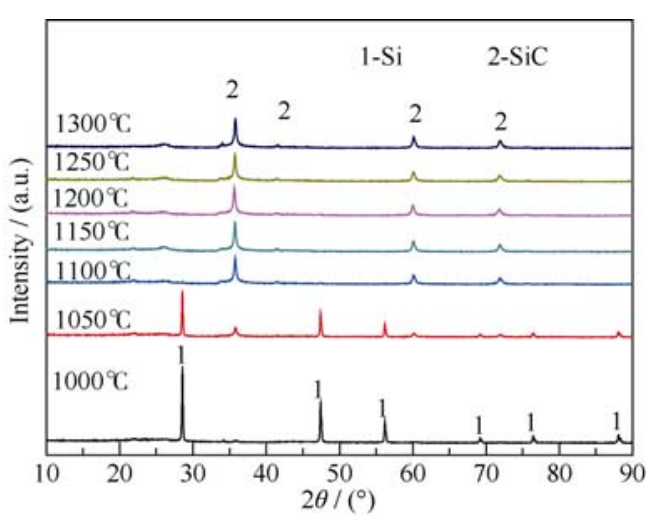

图 $22 \mathrm{wt} \% \mathrm{Ni}$ 为催化剂时, 不同温度反应 $0.5 \mathrm{~h}$ 后试样的 $\mathrm{XRD}$ 图谱

Fig. 2 XRD patterns of samples reacted at various temperatures for $0.5 \mathrm{~h}$ with $2 \mathrm{wt} \% \mathrm{Ni}$ catalysts (Si: JCPDS 01-077-2109; SiC: JCPDS 01-073-1708)

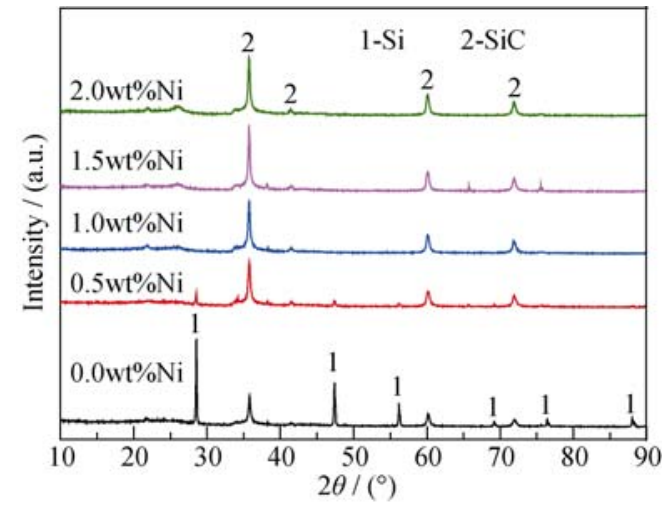

图 3 催化剂 $\mathrm{Ni}$ 加入量不同时, $1150^{\circ} \mathrm{C}$ 反应 $0.5 \mathrm{~h}$ 产物的 $\mathrm{XRD}$ 图谱

Fig. 3 XRD patterns of samples reacted at $1150^{\circ} \mathrm{C}$ for $0.5 \mathrm{~h}$ with various contents of $\mathrm{Ni}$ catalysts (Si: JCPDS 01-077-2109; SiC: JCPDS 01-073-1708)

$1.0 \mathrm{wt} \%$, 单质硅的衍射峰完全消失，说明硅粉已经 完全碳化为碳化硅; 进一步增大催化剂的加入量到 $1.5 \mathrm{wt} \% \sim 2.0 \mathrm{wt} \%$ 时, 产物的物相组成无明显变化。以 上结果表明: 在 $1150^{\circ} \mathrm{C}$ 碳化 $0.5 \mathrm{~h}$ 的条件下, 引入 $1.0 \mathrm{wt} \%$ 的 $\mathrm{Ni}$ 做催化剂即可使硅粉完全碳化生成碳 化硅。

\section{4 以 $\mathrm{Ni}$ 为催化剂时, 反应时间对合成碳化硅的 影响}

当反应温度为 $1150^{\circ} \mathrm{C}$, 催化剂 $\mathrm{Ni}$ 加入量为 $1.0 \mathrm{wt} \%$ 时, 碳化反应不同时间后试样的 XRD 图谱如 图 4 所示。当碳化时间为 $10 \mathrm{~min}$ 时, 试样 XRD 图谱 中依然有很强的硅衍射峰, 说明尚有很多硅粉未参 与碳化反应; 当碳化时间为 $20 \mathrm{~min}$ 时, 单质硅的衍 射峰强度显著下降, 说明大部分硅粉已经转变为碳 化硅; 当碳化时间为 $30 \mathrm{~min}$ 时, 单质硅的衍射峰消 


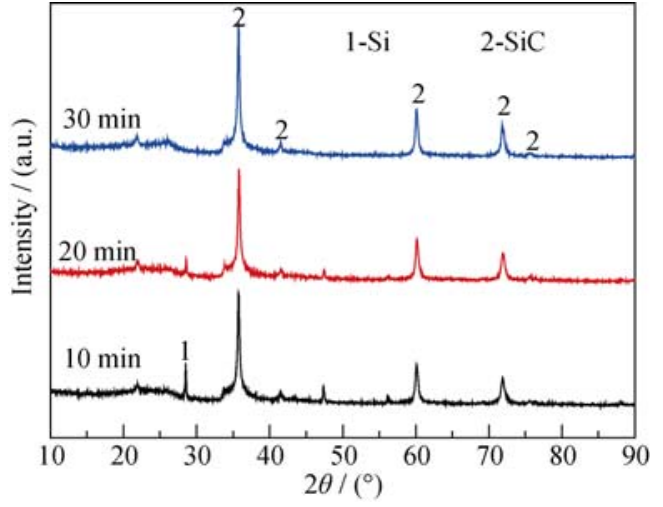

图 4 加入 $1.0 \mathrm{wt} \% \mathrm{Ni}$ 催化剂时, $1150^{\circ} \mathrm{C}$ 反应不同时间后试样 的 XRD 图谱

Fig. 4 XRD patterns of samples reacted at $1150^{\circ} \mathrm{C}$ for various holding time with $1.0 \mathrm{wt} \% \mathrm{Ni}$ catalysts

(Si: JCPDS 01-077-2109; SiC: JCPDS 01-073-1708)

失, 说明单质硅粉已经完全碳化。以上结果表明: 在 本实验条件下，反应 $30 \mathrm{~min}$ 即可使硅粉完全发生碳 化反应转变为碳化硅。与常规加热合成 $\mathrm{SiC}$ 所需的 时间 (2h) 相比 ${ }^{[1,17]}$, 微波加热可使 $\mathrm{SiC}$ 的合成时间 减少约 $1.5 \mathrm{~h}$ 。

\section{5 合成产物的显微结构表征}

图 5 为合成产物的 SEM 照片, 图 5(a)为无催化剂
条件下, $1250^{\circ} \mathrm{C}$ 反应 $0.5 \mathrm{~h}$ 产物的 SEM 照片; 图 5(b) 为加入 $1.0 \mathrm{wt} \%$ 的 $\mathrm{Ni}$ 为催化剂的条件下, $1150^{\circ} \mathrm{C}$ 反应 $0.5 \mathrm{~h}$ 的产物形貌。从图中可以看出, 无论加入催化 剂与否，产物中均有晶须和颗粒两种形貌，其中的 晶须直径约为 $30 \sim 100 \mathrm{~nm}$, 长度可达 $20 \mu \mathrm{m}$ (长径比 约在 50 500 之间), 图 5(b)中点 1 处的 EDS(图 5(b) 中的插图)结果表明，这些晶须应为碳化硅晶须(能 谱中的 $\mathrm{Al}$ 可归因于 SEM 测试时所采用的 $\mathrm{Al}$ 质样 品台, 其中的 $\mathrm{Au}$ 来源于样品表面所喷镀的 $\mathrm{Au}$ 导 电膜)。

为进一步研究所合成碳化硅晶须的显微结构, 采 用 TEM 对所得产物进行了表征。从低倍 TEM 照片(图 6(a)可知，所合成的晶须长度较长。从高倍 TEM(图 6(b) 照片可以看出，晶须的直径约为 30 60 nm。图 6(c) 的 衍射花样表明，所合成的晶须为 $\beta-\mathrm{SiC}$ ，其衍射花样 中存在微弱的零星散落的衍射斑点表明所合成的 $\beta-\mathrm{SiC}$ 晶须中存在着堆积缺陷或孪晶缺陷 ${ }^{[18-19]}$ 。 HR-TEM(图 6(d)结果表明, 晶须的晶格条纹间距约为 $0.25 \mathrm{~nm}$ ，与 $\beta-\mathrm{SiC}(111)$ 晶面方向的晶面间距 $(0.252 \mathrm{~nm})$ 一致, 表明晶须是沿[111]方向生长的。晶格条纹的结 果还表明 $\beta-\mathrm{SiC}$ 晶须中存在很多无规则的堆积缺陷, 这与选区电子衍射的结果基本一致。
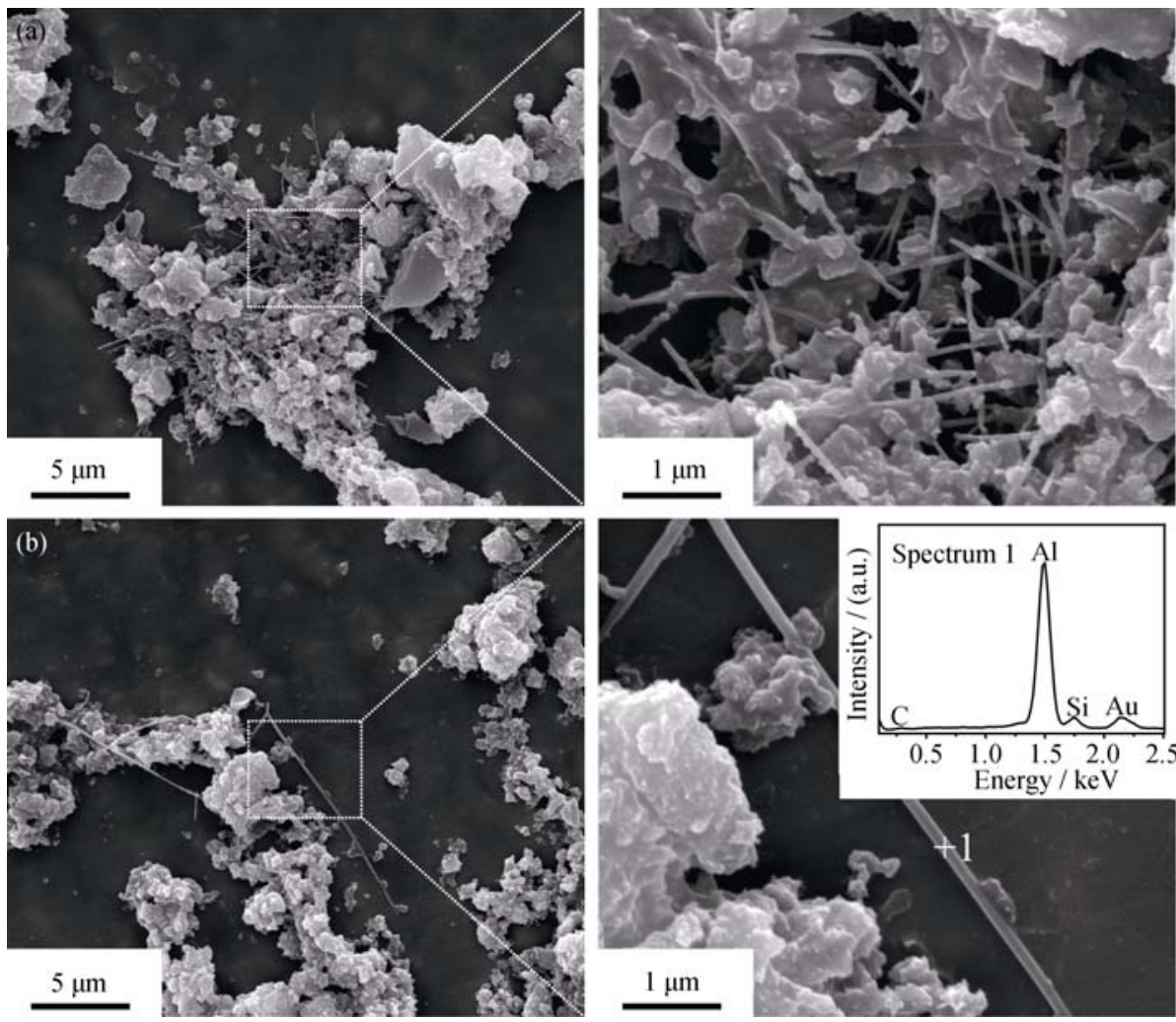

图 5 不同条件下合成 $\beta$-SiC 的 SEM 照片

Fig. 5 SEM images of the as-prepared $\beta$-SiC (a) $1250^{\circ} \mathrm{C} / 0.5 \mathrm{~h}$, without catalyst; (b) $1150^{\circ} \mathrm{C} / 0.5 \mathrm{~h}$, with $1.0 \mathrm{wt} \% \mathrm{Ni}$ as catalyst 

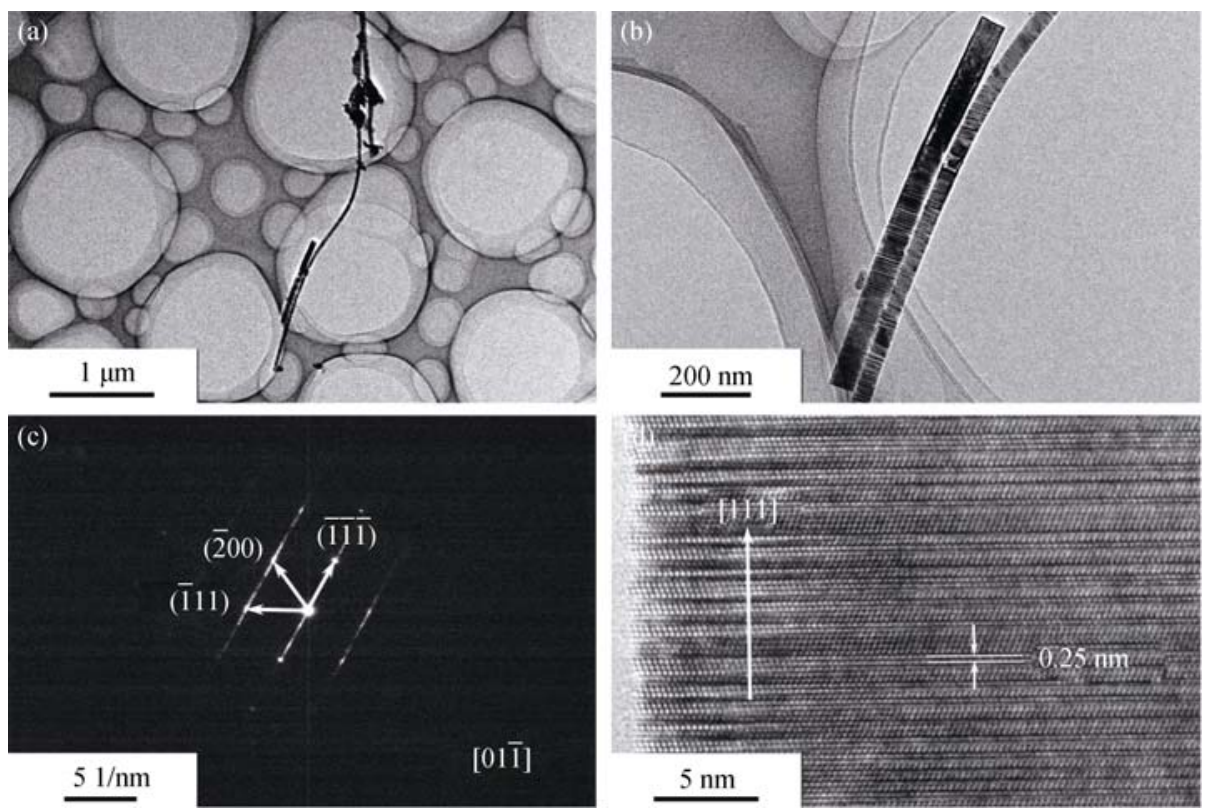

图 6 所合成 $\beta$-SiC 晶须的 TEM((a)低倍, (b)高倍)、SAED (c)和 HR-TEM (d)照片

Fig. 6 TEM ((a) low-and (b) high-magnification), SAED (c) and HR-TEM (d) images of as-prepared $\beta$-SiC whiskers

\section{6 催化剂作用机理分析}

上述研究结果表明, 与不加催化剂时相比, 加 入催化剂 Ni 降低了 $\beta-\mathrm{SiC}$ 的合成温度, 显著促进了 $\mathrm{Si}$ 向 $\beta-\mathrm{SiC}$ 的转化。很多研究表明 ${ }^{[20-21]}$, 采用 $\mathrm{Ni}$ 为 催化剂催化 $\mathrm{Si}$ 粉制备 $\mathrm{SiC}$ 的过程中, $\mathrm{Ni}$ 与 $\mathrm{Si}$ 会首先 形成 Ni-Si 合金, 进而起到催化作用。众所周知, $\mathrm{Si}-\mathrm{Si}$ 键的存在使得 $\mathrm{Si}$ 粉颗粒很难分解为 $\mathrm{Si}$ 原子, 但当 $\mathrm{Si}$ 颗粒与吸附在其表面的 $\mathrm{Ni}$ 形成合金后, $\mathrm{Si}$ 与 $\mathrm{Si}$ 原子 之间的键长可能会被拉长, 从而削弱 $\mathrm{Si}-\mathrm{Si}$ 键的结合 强度，使得其更易与 $\mathrm{C}$ 原子反应生成 $\mathrm{SiC}$ 。

为验证以上推论, 我们以含有 55 个原子的 $\mathrm{Si}$ 纳米团簇 $\left(\mathrm{Si}_{55}\right)$ 和 $\mathrm{Ni}-\mathrm{Si}$ 合金纳米团簇 $\left(\mathrm{Si}_{43} \mathrm{Ni}_{12}\right.$ 和 $\mathrm{Si}_{37} \mathrm{Ni}_{18}$ ) 作为计算模型, 采用 DFT 计算研究了这些 团簇的稳定性。计算结果如表 1 所示。当团簇中仅 有 $\mathrm{Si}$ 原子时 $\left(\mathrm{Si}_{55}\right)$, 其内聚能为 $582.458 \mathrm{~kJ} / \mathrm{mol}$; 当 团簇中掺入 $\mathrm{Ni}$ 原子形成合金时 $\left(\mathrm{Si}_{43} \mathrm{Ni}_{12}\right.$ 和 $\left.\mathrm{Si}_{37} \mathrm{Ni}_{18}\right)$, 其内聚能分别增加至 940.142 和 $1165.307 \mathrm{~kJ} / \mathrm{mol}$, 远高于 $\mathrm{Si}_{55}$ 团簇的内聚能。由文献[22]可知, 内聚能 越大体系越稳定, 这表明, 与单独的 $\mathrm{Si}$ 相比, Ni-Si 合金更稳定，换言之，在催化剂 $\mathrm{Ni}$ 存在的条件下， 很容易形成 Ni-Si 合金。

为进一步研究 $\mathrm{Ni}$ 纳米颗粒催化 $\mathrm{Si}$ 粉生成 $\mathrm{SiC}$ 的
机理，又计算了这些纳米团簇中处于(111)面上边缘位 置的 $\mathrm{Si}$ 原子之间的键长, 结果如图 7 所示。计算结果 表明: 在 $\mathrm{Si}_{55}$ 纳米团簇中, 其(111)面上边缘 $\mathrm{Si}$ 原子之 间的键长为 $0.4619 \mathrm{~nm}$; 在 $\mathrm{Si}_{43} \mathrm{Ni}_{12}$ 合金团簇中, 其边 缘 $\mathrm{Si}$ 原子之间的键长为 $0.4625 \mathrm{~nm}$; 而对 $\mathrm{Si}_{37} \mathrm{Ni}_{18}$ 纳米 团簇而言, 其边缘 $\mathrm{Si}$ 原子之间的键长进一步增长至 $0.4990 \mathrm{~nm}$ 。这表明 $\mathrm{Si}-\mathrm{Ni}$ 合金的形成可使 $\mathrm{Si}$ 原子之间 的键长显著增长, 导致 $\mathrm{Si}$ 原子之间结合强度的削弱, 最终促进了 $\mathrm{Si}$ 原子与 $\mathrm{C}$ 原子之间的反应。

\section{3 结论}

以硅粉和酚醛树脂为起始原料, 以镍为催化剂, 采用微波加热催化反应工艺制备了 $\mathrm{SiC}$ 粉体。其最 佳合成工艺条件如下：反应温度为 $1150^{\circ} \mathrm{C}$, 反应时 间为 $0.5 \mathrm{~h}$, 催化剂 $\mathrm{Ni}$ 的加入量为 $1.0 \mathrm{wt} \%$ 。催化剂 $\mathrm{Ni}$ 的加入可使硅粉的完全碳化温度降低约 $100^{\circ} \mathrm{C}$; 所合成产物中存在着大量晶须状 $\beta-\mathrm{SiC}$, 其直径约为 30 100 nm，晶须沿[111]方向生长。DFT 计算结果表 明，催化剂 $\mathrm{Ni}$ 首先与 $\mathrm{Si}$ 形成 $\mathrm{Ni}-\mathrm{Si}$ 合金，并使得 $\mathrm{Si}$ 原子之间的键长被拉长, 进而促使 $\mathrm{Si}$ 粉在较低温度 下与 $\mathrm{C}$ 发生反应生成 $\mathrm{SiC}$ 。

表 $1 \mathrm{Si}_{55} 、 \mathrm{Si}_{43} \mathrm{Ni}_{12}$ 和 $\mathrm{Si}_{37} \mathrm{Ni}_{18}$ 纳米团簇的总能量和内聚能

Table1 Total energies and cohesive energies of $\mathrm{Si}_{55}, \mathrm{Si}_{43} \mathrm{Ni}_{12}$ and $\mathrm{Si}_{37} \mathrm{Ni}_{18}$ clusters

\begin{tabular}{cccccc}
\hline Clusters composition & Single $\mathrm{Si}$ atom & Single Ni atom & $\mathrm{Si}_{55}$ & $\mathrm{Si}_{43} \mathrm{Ni}_{12}$ & $\mathrm{Si}_{37} \mathrm{Ni}_{18}$ \\
\hline Total energy/(a.u.) & -290.133 & -1390.136 & -15957.557 & -30668.916 & -38024.613 \\
Cohesive energy $/\left(\mathrm{kJ} \cdot \mathrm{mol}^{-1}\right)$ & - & - & 582.458 & 940.142 & 1165.307 \\
\hline
\end{tabular}



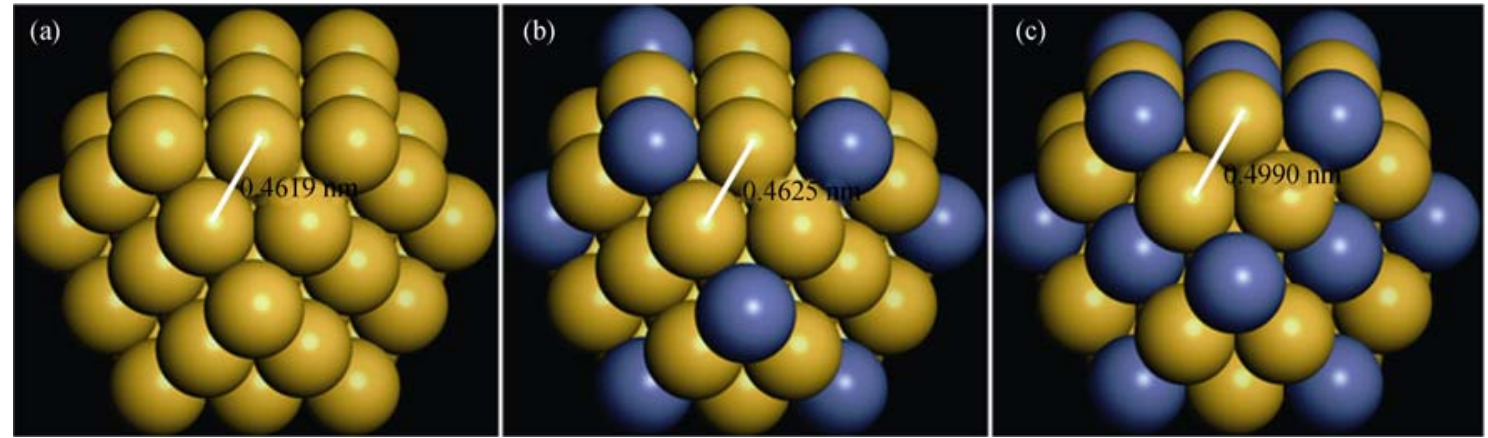

图 7 (a) $\mathrm{Si}_{55}$ 、(b) $\mathrm{Si}_{43} \mathrm{Ni}_{12}$ 和(c) $\mathrm{Si}_{37} \mathrm{Ni}_{18}$ 纳米团簇中(111)面上边缘位置 $\mathrm{Si}$ 原子间的键长计算结果,

Fig. 7 The DFT calculations of bond length of $\mathrm{Si}$ atoms at edge sites0 on (111) face of $\mathrm{Si}_{55}(\mathrm{a}), \mathrm{Si}_{43} \mathrm{Ni}_{12}$ (b) and $\mathrm{Si}_{37} \mathrm{Ni}_{18}$ (c) clusters

\section{参考文献:}

[1] SHI L, ZHAO H, YAN Y, et al. Synthesis and characterization of submicron silicon carbide powders with silicon and phenolic resin. Powder Technology, 2006, 169(2): 71-76.

[2] CAO Y, ZHANG H, LI F, et al. Preparation and characterization of ultrafine $\mathrm{ZrB}_{2}-\mathrm{SiC}$ composite powders by a combined Sol-Gel and microwave boro/carbothermal reduction method. Ceramics International, 2015, 41(6): 7823-7829.

[3] HUANG SHAN, WANG JI-GANG, LIU SONG, et al. Structural characterization and photoluminescence properties of SiC nanowires prepared by microwave method. New Carbon Materials, 2015, 30(3): 230-235.

[4] ZHANG H, LI F. Preparation and microstructure evolution of diboride ultrafine powder by Sol-Gel and microwave carbothermal reduction method. Journal of Sol-Gel Science and Technology, 2008, 45(2): 205-211.

[5] ZHANG H, LI F, JIA Q, et al. Preparation of titanium carbide powders by sol-gel and microwave carbothermal reduction methods at low temperature. Journal of Sol-Gel Science and Technology, 2008, 46(2): 217-222.

[6] ZHANG H, LI F, JIA Q. Preparation of titanium nitride ultrafine powders by Sol-Gel and microwave carbothermal reduction nitridation methods. Ceramics International, 2009, 35(3): 1071-1075.

[7] DENG X, DU S, ZHANG H, et al. Preparation and characterization of $\mathrm{ZrB}_{2}-\mathrm{SiC}$ composite powders from zircon via microwave-assisted boro/carbothermal reduction. Ceramics International, 2015, 41(10): 14419-14426.

[8] LI F, FU F, LU L, et al. Preparation and artificial neural networks analysis of ultrafine $\beta$-Sialon powders by microwave-assisted carbothermal reduction nitridation of Sol-Gel derived powder precursors. Advanced Powder Technology, 2015, 26(5): 1417-1422.

[9] HUANG Z, DENG X, LIU J, et al. Preparation of $\mathrm{CaZrO}_{3}$ powders by a microwave-assisted molten salt method. Journal of the Ceramic Society of Japan, 2016, 124(5): 593-596.

[10] PENG HU, LI JUN. Advance in microwave high-temperature heating technology. Materials Review, 2005, 19(10): 100-103.
[11] LU BIN, LIU JI-XUAN, ZHU HUA-WEI, et al. SiC nanowires synthesized by microwave heating. Journal of Inorganic Materials, 2007, 22(6): 1135-1138.

[12] FU FANG, JIA XIAO-LIN, ZHANG HAI-JUN. Synthesis of SiC ultrafine powder by Sol-Gel and microwave carbothermal reduction method. Rare Metal Materials and Engineering, 2008, 37(S1): 153-155.

[13] HAO BIN. Study on preparation of SiC powder by microwave sintering. Bulletin of The Chinese Ceramic Society, 2015, 34(3): 864-867.

[14] YAN XIAO-YAN, WANG YING-YONG, JIN GUO-QIANG. Preparation and characterization of $\mathrm{SiC}$ nanowires with $\mathrm{Zr}$ as catalyst. Silicone Material, 2011, 25(5): 297-299.

[15] WANG D, XU D, WANG Q, et al. Periodically twinned SiC nanowires. Nanotechnology, 2008, 19(21): 2386-2390.

[16] XU WU-JUN, XU YAO, SUN XIAN-YONG, et al. Fabracation of tower like $\beta$-SiC by Sol-Gel and carbothermal reduction processing. New Carbon Materials, 2006, 21(2): 167-170.

[17] ZHAO H, SHI L, LI Z, et al. Silicon carbide nanowires synthesized with phenolic resin and silicon powders. Physica E: Low-dimensional Systems and Nanostructures, 2009, 41(4): 753-756.

[18] LI Q, WANG C A, SHENGNIAN T. Synthesis of bamboo-like SiC whiskers from waste silica fume. Crystal Research \& Technology, 2014, 49(5): 290-297.

[19] LU M, LI A, WANG T, et al. Synthesis of bamboo-like 3C-SiC nanowires by microwave assisted carbothermal reduction. Journal of Nanoscience \& Nanotechnology, 2010, 10(3): 2135-2138.

[20] ZHANG J, LIU X, JIA Q, et al. Novel synthesis of ultra-long single crystalline $\beta$-SiC nanofibers with strong blue/green luminescent properties. Ceramics International, 2016, 42(3): 4600-4606.

[21] XIA ZHONG-FENG, WANG ZHOU-FU, WANG XI-TANG, et al. In-situ formation of silicon carbide whisker by Ni-catalyzed silicon-modified pitch and its mechanism. Refractories, 2014, 48(4): 254-256.

[22] ZHANG H, LU L, KAWASHIMA K, et al. Synthesis and catalytic activity of crown jewel-structured (IrPd)/Au trimetallic nanoclusters. Advanced Materials, 2015, 27(8): 1383-1388. 